\title{
Patterns of meningococcal infection in Sudan with emergence of Neisseria meningitidis serogroup W135
}

\author{
M.S. Karsany, ${ }^{7}$ A.A. Elshayeb, ${ }^{7}$ E.S. Saeed, ${ }^{2}$ R. Elaagib, ${ }^{3}$ S.A. Ibrahim, ${ }^{4}$ E. Elsamani, ${ }^{5}$ K. Hussien ${ }^{6}$ and K.M. Salih ${ }^{7}$
}

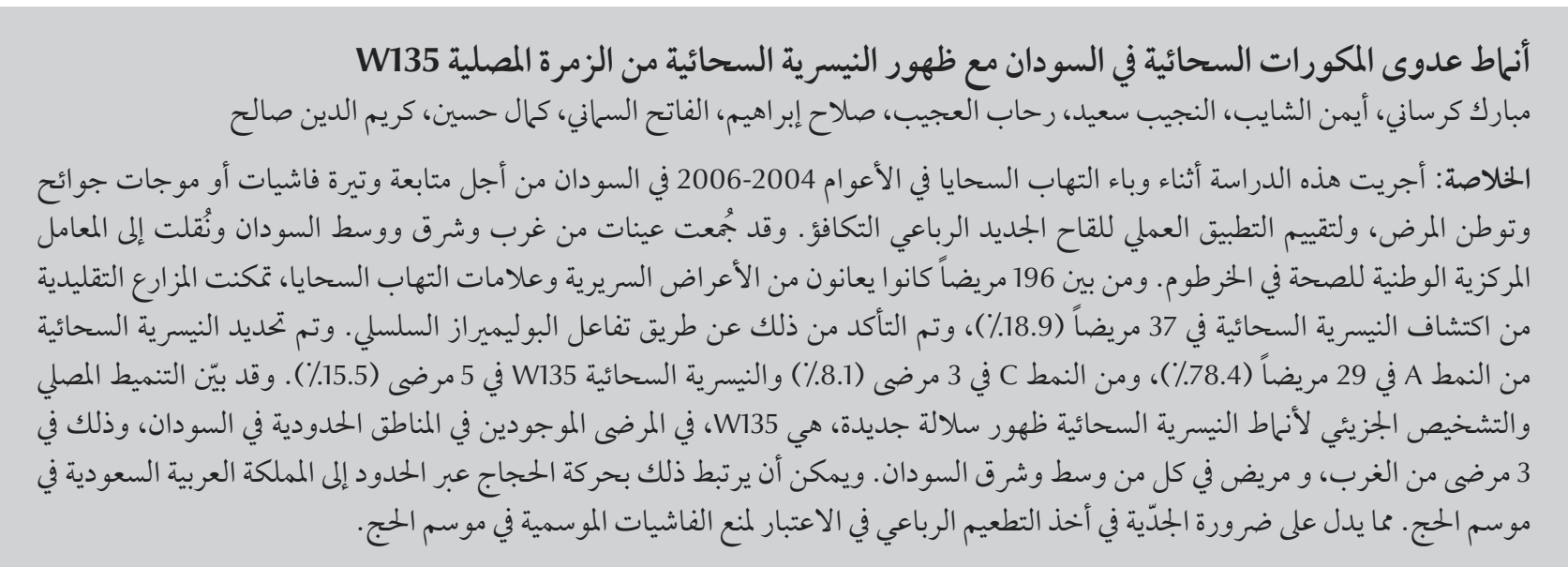

ABSTRACT This study was conducted during the 2004-2006 epidemic of meningitis in Sudan to follow-up the frequency of disease outbreak or endemic waves and to evaluate the new quadrivalent vaccine for actual application. Samples were collected from Darfur, El Gedaref, Kassala and Khartoum States and transported to the National Health Central Laboratory in Khartoum. Of 196 patients with clinical symptoms and signs of meningitis, conventional culture identified Neisseria meningitidis in 37 (18.9\%), confirmed by polymerase chain reaction. $N$. meningitidis type A was identified in 29 (78.4\%) patients, type C in 3 (8.1\%) and N. meningitidis W135 in 5 (15.5\%). The serotyping and molecular diagnosis patterns of $N$. meningitidis showed the emergence of the new strain, W 135, in patients from the borders of Sudan, 3 from the West Darfur, and 1 each from El Gedaref and Kassala. These could be related to the movement of pilgrims through the borders to Saudi Arabia in the Hajj season. Serious consideration may be needed for quadrivalent vaccination to prevent seasonal and Hajj season outbreaks.

Tableau des infections à méningocoques au Soudan avec l'émergence du sérogroupe W135 de Neisseria meningitidis

RÉSUMÉ La présente étude a été réalisée pendant l'épidémie de méningite de 2002-2004 au Soudan pour surveiller la fréquence des flambées de maladie ou les vagues endémiques et pour évaluer le nouveau vaccin quadrivalent en vue d'une application concrète. Des échantillons ont été collectés dans les États du Darfour, d'El Gedaref, de Kassala et de Khartoum et transportés au laboratoire national de santé central à Khartoum. La culture de type classique a permis d'identifier Neissera meningitidis chez 37 (18,9 \%) des 196 patients ayant des symptômes et des signes cliniques de méningite, ce qui a ensuite été confirmé par réaction en chaîne par polymérase. $N$. meningitidis de type $\mathrm{A}$ et $\mathrm{C}$ a été identifié respectivement chez $29(78,4 \%)$ et 3 patients $(8,1 \%)$ et $N$. meningitidis W135 chez 5 patients (15,5\%). Les caractéristiques du sérotypage et du diagnostic moléculaire de N. meningitidis ont montré l'émergence d'une nouvelle souche, W 135, chez les patients venant des états frontières du Soudan, 3 du Darfour occidental, et 2 respectivement d'El Gedaref et de Kassala. Ces cas pourraient avoir un lien avec le déplacement des pèlerins qui traversent les frontières pour se rendre en Arabie saoudite durant la saison du Hajj. La vaccination par le vaccin quadrivalent devrait être sérieusement prise en compte pour prévenir les flambées épidémiques saisonnières et liées à la saison du Hajj.

'Department of Microbiology, College of Medical Laboratory Sciences, Karary University. Khartoum, Sudan (Correspondence to A.A. Elshayeb: ayman_elshayeb@yahoo.com. ${ }^{2}$ Department of Microbiology; ${ }^{4}$ Department of Paediatrics, Faculty of Medicine, Khartoum University, Khartoum, Sudan. ${ }^{3}$ National Health Laboratory, National Ministry of Health, Khartoum, Sudan. ${ }^{5}$ Department of Community Medicine. Ahfad University for Women, Khartoum, Sudan. ${ }^{6}$ Faculty of Medicine, Ribat University, Khartoum, Sudan. ${ }^{7}$ Department of Paediatrics, Gaafar Ibn Aouf Children's Hospital, Bahri University, Khartoum, Sudan.

Received: 17/01/12; accepted: 22/05/12 


\section{Introduction}

Meningococcal disease is has high mortality and morbidity rates and should be notified to health authorities [1]. Neisseria meningitidis is a common cause of serious illness such as septicaemia and meningitis in both children and adults $[2,3]$. The pattern of the disease in developing countries is in the form of outbreaks, particularly in Africa, unlike the sporadic patients seen in developed countries [4]. Exploitation of the immunological reaction to the capsular polysaccharides has lead to the identification of about 13 subgroups, of which $\mathrm{A}, \mathrm{B}$ and $\mathrm{C}$ are considered the main pathogens [4].

Historically, the meningococci causing the 1906-1908 outbreak were possibly introduced by pilgrims returning from Mecca and pilgrims were also implicated in an epidemic in Sudan a few years earlier [5]. Subsequently, 3 epidemic waves began in 1934, 1942 and 1949, the last resulting in over 50000 patients in 1950 and 1951 in Sudan and Nigeria and 16000 patients in Burkina Faso in 1957.

Virtually all bacterial isolates obtained in the first decades of the 20th century showed serogroup A capsule agglutination except for a few isolates from Sudan where serogroup B was seen or the serogroup could not be determined with the available antisera [6]. Recently, new subgroups like the $\mathrm{Y}$ and W135 meningococci have been recorded as causing severe disease $[7,8]$.

The disease is highly endemic in African countries in a large area extending all the way from Gambia in the west, to Sudan in the east which has been known as the meningitis belt [9]. The first report of recognition of new subgroup due to W135 occurred in 2000 during the Hajj season in Saudi Arabia [10]. The first time W135 was detected by Issa et al. in the Eastern part of Sudan in the year after the 2000
Hajj season [11]. Many European and African countries have used a bivalent A\&C meningococcal polysaccharide vaccine for outbreak control and travel immunization, whereas others (the United States of America, Canada, Italy, Portugal, Denmark, and Saudi Arabia) routinely use the quadrivalent (A, C, Y, and W135) preparation. In the United Kingdom and France, the quadrivalent vaccine is used only for prophylaxis among contacts of proven serogroup Y or W135 disease patients [11].

Since meningitis is a serious disease in Sudan during summer seasons, the overall objective of this study was to follow-up the frequency of outbreaks and pan-endemic waves and to evaluate the new quadrivalent vaccine for actual application.

\section{Methods}

Samples of cerebrospinal fluid (CSF) were collected from patients in Darfur, Khartoum, Gadarif and Kassala States in Sudan during the epidemic meningitis season, summers of 2004-2006. The patient definition of meningitis is acute onset of fever, refusal of feeding, vomiting, convulsions, bulging fontanelles and neck stiffness plus signs of meningeal irritation; positive Kerning's sign, Brudziniski sign and/or tripod sign $[12,13]$. All adults and children admitted to hospitals in western, eastern and central Sudan with suspected clinical diagnosis of meningitis were included in the study. Children who were comatose, critically ill or whose guardians did not give consent were excluded. Consent was refused for 21 (20\%) children in total.

After obtaining informed verbal consent, a pre-coded questionnaire on medical, social and family history was completed by the physician and recorded in the hospital statistical department. A thorough clinical examination of each patient was then conducted; CSF was withdrawn from those suspected of meningitis, and examined for macroscopic appearance, cytology, Gram stain, sugar, protein and serology. We retained $4 \mathrm{~mL}$ of the CSF for culture in transport media; this was transported to the National Health Central Laboratory in Khartoum. The samples were subcultured in GCII chocolate agar (Oxoid Limited Hampshire-United Kingdom), and incubated for 24-72 hours in $10 \% \mathrm{CO}_{2}[14]$ atmospheric pressure at $37^{\circ} \mathrm{C}$. Chemical tests were carried out including Gram stain, catalase, oxidase, and Haemophilus analytic profile index (API NH, bioMérieux, Marcy l'Etoile, France) to differentiate between Haemophilus influenzae type $b$ and $N$. meningitidis since both cause meningitis. Serotyping was done using specific antisera (Oxoid Agglutinating Sera) for identification of $H$. influenzae type b, N. meningitidis by rapid slide agglutination.

Quality control was performed using standard control reagents for the media used and for Gram staining according to Clinical and Laboratory Standards Institute guidelines [15].

\section{Results}

Between April 2004 and December 2006, 196 patients with suspected bacterial meningitis according to the patient definition were enrolled in the study. During the epidemic, the age distribution shifted from young children to teenage children and young adults and the attack rates increased.

The 196 patients with suspected meningitis in the study group included 108 paediatric patients ( $\leq 15$ years old) (55\%) and $88(45 \%)$ adults. The sex distribution shows a male predominance, 117 (65\%) males and 69 (35\%) females.

The dominance of $N$. meningitidis among the species isolated, even taking 
into account the contamination and negative growth, was obvious. The types of organisms isolated from the CSF samples from the 196 patients in the study group showed a diversified patterns of isolates: $N$. meningitidis 37 isolates (18.9\%), H. influenzae 9 isolates (4.6\%) and Streptococcus pneumoniae 5 isolates (2.6\%). In 80 (40.8\%) samples there was no growth of organisms and 65 (33.1\%) were contaminated.

The confirmation of results by polymerase chain reaction (PCR) testing also showed the dominance of N. meningitidis. This was carried out on the 145 samples in which there was either contamination or no growth. $N$ meningitidis was identified in 41 samples (28.2\%), H. influenzae in 13 (9.2\%) and S. pneumoniae in 5 (7.6\%). Results were negative in 80 (55.20\%) samples. Samples testing positive by PCR were identified among all age groups, while Hib PCR-positivity was only identified in patients in the under 5-years age group.

N. meningitidis type A was identified in 29 (78.4\%) patients, type C in $3(8.1 \%)$ and N. meningitidis W135 in $5(15.5 \%)$. The determination patterns of $N$. meningitidis showed the emergence of new strains of W135 in patients from the borders of Sudan, 3 from the West, and 1 each from Central and East Sudan. The emergence of N. meningitidis W135 was identified by serology and found in patients from the border states (West Darfur, Kassala and Elgedaref).

\section{Discussion}

The purpose of this study was to determine patterns of $N$. meningitidis distribution and the probabilities of new serotype emergence in different parts of Sudan. During the epidemic period it was obvious that the disease spread among adults more than children $\leq 15$ years in a very odd phenomenon of infection. This has been observed in Scotland [16], Canada [17], and the United States of America [18], where similar increases in meningococcal disease, with a shift to infection of older age groups, have been reported.

More males were infected than females; this is probably related to the daily exposure time to transmission factors and to active movement through borders. However, Sudan is in the African meningitis belt where N. meningitidis is endemic, and this may also explain more adults being affected, despite the herd immunity.

The utilization of molecular biology tools (PCR) in the daily routine diagnosis enhanced the probabilities of detecting and characterizing the different serotypes of meningococcal species. It is evident that N. meningitidis was the most common cause of meningitis, mainly due to N. meningitidis type A. This was in keeping with the results of numerous other studies carried out in Sudan and other developing countries during the same epidemic episodes [19-21]. In fact, a great deal of data has confirmed $N$. meningitidis group $\mathrm{A}$ has the tendency to occur in epidemic forms [19]. Although most of the serotypes of $N$. meningitidis are of types $A$ and $C$ in Africa, in this study W135 was demonstrated in 5 samples $(13.5 \%)$. The emergence of this W135 in Sudan is in keeping with the results of other research in Africa [22] as well as in other parts of the world such as China [23].

The emergence of W135 through the eastern and western borders to the centre of Sudan could be a result of the movement of pilgrims and citizens from West Africa to the Hajj through the eastern part of Sudan to Saudi Arabia. The identification of serotype W135 outbreaks in Sudan is in agreement with previous reports, which indicated that many African countries were affected after the 2000 Hajj season $[24,25]$.

In conclusion, it was clear that control measures on borders and careful investigations with sensitive and specific tools can increase our knowledge towards the recognition of $N$. meningitidis strains. This can improve our awareness of prevention and treatment. This has been observed in countries like the United States of America, Canada, etc. where quadrivalent vaccine against N. meningitidis is used for prophylaxis: no patients with the W135 strain were reported [24]. In Sudan quadrivalent instead of bivalent vaccine was adopted first in Darfur refugee camps, then it was recommended to be given to hajj pilgrims by the Hajj Health Authorities in 2006 and then in the whole of Sudan by 2007 although meticulous surveillance indicate that no critical infections have been recorded clinically to date.

\section{Acknowledgements}

The authors would like to acknowledge the great help given by WHO EMRO Sudan Office for the provision of diagnostic kits and facilitation of the transportation of samples to the Central Laboratory, especially through the early-warning system for Darfur. We are indebted to the Epidemiology Department of the General Directorate of Public Health, Federal Ministry of Health for part of the surveillance data. Our thanks are also extended to the physicians, nurses and laboratory personnel for their excellent assistance.

Funding: This work was funded through the routine laboratory-based surveillance which has been carried out annually since 2004.

Competing interests: None declared. 


\section{References}

1. Bae SM, Kang YH. Serological and genetic characterization of meningococcal isolates in Korea. Japanese Journal of Infectious Diseases, 2008, 61:434-437.

2. Public Health Laboratory Service. Guidelines for public health management of meningococcal disease in the UK. Public Health, 2002, 5:187-204.

3. Rosenstein NE et al. The changing epidemiology of meningococcal disease in the United States, 1992-1996. Journal of Infectious Diseases, 1999, 180:1894-1901.

4. Control of epidemic meningococcal disease, WHO Practical Guidelines, 2nd ed. Geneva, World Health Organization, 1998.

5. Greenwood B. Manson Lecture. Meningococcal meningitis in Africa. Transactions of the Royal Society of Tropical Medicine and Hygiene, 1999, 93:341-353.

6. Lapeyssonnie L. La méningite cérébro-spinale en Afrique [Cerebrospinal meningitis in Africa]. Bulletin of the World Health Organization, 1963, 28(Suppl.):1-114.

7. Vedros NA. Development of meningococcal serogroups. In: Vedros NA, ed. Evolution of Meningococcal Disease, vol. 11. Roes Raton, Florida, CRC Press Inc., 1987:33-37.

8. Manchanda V, Gupta S, Bhalla P. Meningococcal disease: history, epidemiology, pathogenensis, clinical manifestation, diagnosis, antimicrobial susceptibility and prevention. Indian Journal of Medical Microbiology, 2006, 24:7-19.

9. Christopher BS. A molecular epidemiological analysis of meningococcal isolates within Scotland 1972-1998 [thesis]. Glasgow, Institute of Biomedical Life Sciences, University of Glasgow, December 2008.

10. Balkhy HH, Memish ZA, Osoba AO. Meningococcal carriage among local inhabitants during pilgrimage 2000. International Journal of Antimicrobial Agents, 2001, 2003:107-111.

11. Issa M et al. Nesseria meningitidis serotype W-135 isolated from healthy carriers and patients in Sudan after the Hajj 2000. Scandinavian Journal of Infectious Diseases, 2003, 35(4):230233.

12. Hill DJ et al. Cellular and molecular biology of Neisseria meningitidis colonization and invasive disease. Clinical Science, 2010, 118:547-564.
13. Scheupner CJ, Sosnowski KM. Infections caused by Haemophilus and Bordetella species. In: Kelley WN, ed. Textbook of Internal Medicine, 3rd ed. New York, Lippincott-Raven, 1997:1659-1661.

14. The childhood seizures of coma. In: Segar M, ed. Tropical paediatrics. London, Cambridge University Press, 2000:311-312.

15. Performance Standards for Antimicrobial Susceptibility Testing; M100-S11. Wayne, Pennsylvania, Clinical and Laboratory Standards Institute, 2001.

16. Fallon RJ. Meningococcal infections in Scotland. Journal of Medical Microbiology, 1988, 26:168-172.

17. Whalen $\mathrm{CM}$ et al. The changing epidemiology of invasive meningococcal disease in Canada, 1985 through 1992. Emergence of a virulent clone of Neisseria meningitidis. Journal of the American Medical Association, 1995, 273:390-394.

18. Jackson LA et al. Serogroup C meningococcal outbreaks in the United States. An emerging threat. Journal of the American Medical Association, 1995, 273:383-389.

19. Uchiumi $\mathrm{F}$ et al. The effect of resveratrol on the Werner syndrome RecQ helicase gene and telomerase activity. Current Aging Science, 2011, 4(1):1-7.

20. Bart A et al. The population structure of Neisseria meningitidis serogroup A fits the predictions for clonality. Infection, Genetics and Evolution, 2001, 1(2):117-122.

21. Salih MA et al. Characterization of epidemic and nonepidemic Neisseria meningitidis serogroup A strains from Sudan and Sweden. Journal of Clinical Microbiology, 1990, 28:1711-1719.

22. Sié A et al. ST2859 serogroup A meningococcal meningitis outbreak in Nouna Health district, Burkina Faso: a prospective study. Tropical Medicine \& International Health, 2008, 13(6):861-868.

23. Chen $\mathrm{C}$ et al. A first meningococcal meningitis case caused by serogroup X Neisseria meningitidis strains in China. Chinese Medical Journal, 2008, 121:664-666.

24. Emergence of W735 meningococcal disease. Report of a WHO consultation, Geneva, 17-18 September 2001. Geneva, World Health Organization, 2002(WHO/CDS/CSR/GAR/2002.1).

25. Taha MK et al. Serogroup W135 meningococcal disease in Hajj pilgrims. Lancet, 2000, 356(9248):2159. 